\title{
Reliability Analysis of Bonded Joints with Variations in Adhesive Thickness
}

\author{
Jean-Denis MATHIAS* \\ IRSTEA, \\ Laboratoire d'Ingénierie pour les Systèmes Complexes, \\ Campus des Cézeaux, 24 Avenue des Landais - BP 50085, 63172 Aubière Cedex, France \\ Maurice LEMAIRE \\ Clermont Université, Institut Français de Mécanique Avancée, \\ EA 3867,Laboratoire de Mécanique et Ingénieries, \\ Campus de Clermont-Ferrand les Cézeaux - BP265, 63175 AUBIERE Cedex, France
}

Short Title: Reliability analysis of bonded joint

*Corresponding author: email:jean-denis.mathias@,irstea.fr, tel: +33(0)473440680, fax : 33.(0)4.73.44.06.96 


\begin{abstract}
Bonded joints are used in several industrial applications as a surrogate of more expensive repairs, but their reliability must be ascertained. Failure in a bonded joint mainly occurs in the adhesive due to stress concentrations that directly depend on the adhesive thickness. In practice, it is difficult to ensure a good accuracy of the final adhesive thickness, leading to uncertainty to its spatial variability. This uncertainty greatly influences the strength of the bonded joint. This work deals with one of the main key-issues in bonded joints: the influence of the spatial variations in the adhesive thickness on the reliability of the joint; an excessive shear stress level caused by the adhesive thickness variations may lead to failure. This paper provides reliability analysis by considering the adhesive thickness as a stochastic field. The experimental thickness field is obtained so as to identify the stochastic parameters. These parameters are then introduced in a structural reliability model to evaluate the failure probability. Results show the influence of adhesive thickness uncertainty on bonded joint failure.
\end{abstract}

KEYWORDS: bonded joint, failure, shear stress, adhesive thickness, stochastic field, reliability. 
Author produced version of the paper published in Journal of Adhesion Science and technology, vol. 27 (10), p. 1069-1079., 2013 Original publication available at http://www.tandfonline.com,

DOI : $10.1080 / 01694243.2012 .727176$

\section{Introduction}

Bonded composite patches are used as structural repairs in several fields such as civil engineering for damaged concrete structures [1] or aeronautics for components which exhibit damages, defects or impacts [2]. Another industrial application consists in using patches for the prevention of damage appearance. The service life of such reinforced structures is expected to be increased and expensive repairs or replacements are also expected to be avoided. However, it is well known that shear stress peaks near the free edges of the composite patches may cause failure of the joint. Indeed, some studies have shown that 53\% of significant defects on bonded structures proceed from adhesive bond failures [3].

The shear-lag model was the first model which enabled the calculation of stress distribution in the adhesive, and consequently the prediction of bonded joint failure [4]. This model was then refined to account for some additional factors such as spew fillet [5], large deflections [6] or possible elastic-plastic response of the adhesive [7,8]. Some other nonlinear models have been developed, for instance to take into account the viscoelasticity of the adhesive [9] or the influence of the load level on the parameters of the Burgers model [10]. It is well known that the adhesive thickness greatly influences the shear stress distribution. Several studies have shown the influence of this parameter. For example, cohesive zone models have been used to analyze the influence of the adhesive thickness [11]. A comparative numerical and experimental study has also been done for aluminum single-lap joints bonded with aluminum powder filled epoxy adhesive [12]. The type of failure according to the adhesive thickness has been also studied [13].

It is commonly admitted that adhesive thickness presents some uncertain variations owing to the joint fabrication process. These variations can significantly increase the shear stress peak near the free edge of the joint. Reliability methods [14] can take into account this variability by considering the adhesive thickness as a stochastic field. Therefore, it enables the assessment of the failure probability of the bonded joint. Linear or quadratic approximations of the limit state (characterizing the bonded joint failure) or response surface approximations can be used for the calculation of this failure probability avoiding a too high number of simulations such as classical Monte Carlo simulations [14] [15]. It has been successfully applied in several studies, especially in mechanical applications [16] [17].

An experimental investigation of adhesive thickness variations is first addressed in this paper. For this purpose, a three-dimensional measuring machine is used in order to obtain the adhesive thickness field of bonded joint. The stochastic parameters of the adhesive thickness field are then identified by a stochastic decomposition. Afterwards, these parameters are introduced in a structural reliability model to calculate failure probabilities and normalized sensitivities with respect to the mean [14]. Finally, the reliability model is used to calculate the safety coefficient for a target failure probability.

\section{Experimental assessment of the adhesive}

\subsection{Experimental set-up}

Single-lap specimens have been prepared by the industrial partner, "AIA of ClermontFerrand" (French Ministry of Defence). Composite patches were bonded to aluminium (see Figure 1). The substrate was made of aluminium $2024 \mathrm{~T} 3$ with a thickness $4 \mathrm{~mm}$. The composite patch was made of Hexcel carbon prepreg system 914 T300 (epoxy/carbon). Four 
unidirectional plies were used. The total thickness of the patch was $0.5 \mathrm{~mm}$. The dimensions of the composite patch were equal to $L \mathrm{x} L$ with $L=70 \mathrm{~mm}$. The adhesive used was a Redux 312 adhesive. The initial setting of the adhesive thickness during the joint fabrication process was $0.15 \mathrm{~mm}$. Mechanical properties of each material are reported in Table 1.

As mentioned above, the adhesive thickness may present some uncertain variations owing to the joint fabrication process. Before identifying the stochastic parameters, the adhesive thickness field has to be measured. For this purpose, a three-dimensional measuring machine (MMT TEMPO MCA 10 from TRI-MESURES, France) was used here to obtain the thickness field of the adhesive with respect to the $x$ - and $y$-directions. The resolution was 5 $\mu \mathrm{m}$. Measurements were made on both faces of the specimen. The pitch $u$ (the distance between two measures) here was $1.1 \mathrm{~mm}$ which corresponds to 64 pitches for the specimen length in the $x$-direction. 37 pitches were used for the specimen width in the $y$-direction. The path of the feeler of the three-dimensional measuring machine is represented in Figure 1. The total thickness $t^{\text {tot }}(x, y)$ was then obtained. The composite thickness $t^{\text {comp }}(x, y)$ and the aluminium thickness $t^{\text {alu }}(x, y)$ were assumed to be constant. The adhesive thickness $t^{\text {adh }}(x, y)$ can be determined as follows:

$$
t^{a d h}(x, y)=t^{t o t}(x, y)-t^{a l u}(x, y)-t^{c o m p}(x, y)
$$

Note that variations in thicknesses of the aluminium plate $t^{a l u}(x, y)$ and of the composite $t^{\text {comp }}(x, y)$ were negligible in comparison with the adhesive thickness variations.

\subsection{Field decomposition}

The adhesive thickness $t^{a d h}(x, y)$ is now considered as a stochastic field $t^{a d h}(x, y, \omega)$ in which $\omega$ are random points in space. In the following, we consider a one-dimensional mechanical model based on the Volkersen's model [4] (see Section 4.1) in the $x$-direction. This model has some limitations and more complex and accurate models could be used. However, this model is commonly used in the bonded joint community. Therefore, for the sake of simplicity and to show the feasibility of the current approach, this model is chosen here. The $x$-direction corresponds to the fiber direction of the composite patch and to the direction of the loading (see section 4.1). This leads to consider the stochastic properties of the stochastic field only along the $x$-direction. In this case, we consider the stochastic adhesive thickness field along the $y$-direction $t^{a d h}(x, y, \omega)$ as 37 experimental realizations named "replicas" (37 pitches were used for the specimen width in the $y$-direction) with 64 points per replica (in the $x$-direction). Each adhesive thickness replica $i$ depends only on the $x$ direction and is denoted as $t_{i}^{a}\left(x, \omega=\omega_{i}\right)_{1 \leq i \leq 37}$. We consider these experimental replicas $t_{i}^{a}\left(x, \omega=\omega_{i}\right)_{1 \leq i \leq 37}$ as realizations of the stochastic field $t^{a}(x, \omega)$ that we want to characterize. The thickness field of the adhesive $t^{a}(x, \omega)$ presents two types of variations. The first variation is global and can be represented by a mean deterministic field $t^{\text {mean }}(x)$ which can be modelled by a polynomial function. The second variation is modelled with a stochastic field $Y(x, \omega)$, which leads to:

$$
t^{a}(x, \omega)=t^{\text {mean }}(x)+Y(x, \omega)
$$


For the identification procedure of $t^{\text {mean }}(x)$ and $Y(x, \omega)$, we use the experimental replicas $i$. For each replica $i$, we have the same decomposition:

$$
t_{i}^{a}\left(x, \omega=\omega_{i}\right)=t_{i}^{\text {mean }}(x)+Y_{i}\left(x, \omega=\omega_{i}\right)
$$

For each replica $i$, the aim is to extract a trajectory $Y_{i}\left(x, \omega=\omega_{i}\right)$ that has a mean equal to 0 (see Figure 2).

Decomposition of the adhesive thickness is represented in Figure 3. Each replica $t_{i}^{a}\left(x, \omega=\omega_{i}\right)$ is smoothed leading to the mean deterministic field $t_{i}^{\text {mean }}(x)$. Residuals are then obtained by subtracting the mean deterministic field from the full thickness field and are considered as the trajectory $Y_{i}\left(x, \omega=\omega_{i}\right)$, with a mean equal to 0 . We can see that the mean deterministic field $t_{i}^{\text {mean }}(x)$ decreases near the free edge of the patch. This is due to the joint fabrication process in this case. This deterministic decrease in the adhesive thickness leads to a stress concentration within the adhesive near the free edge. Uncertain variations may increase this stress concentration. The aim then is to determine the stochastic parameters of the stochastic field $Y(x, \omega)$ from replica trajectories $Y_{i}\left(x, \omega=\omega_{i}\right)$ and to introduce them in a reliability model.

\section{Identification of the $Y(x, \omega)$ stochastic field parameters}

\subsection{Assumptions}

Before beginning the identification, some assumptions regarding $Y(x, \omega)$ must be made. $Y(x, \omega)$ is supposed to be a stationary second-order process. As explained above, experimental data are considered as realizations of $Y(x, \omega)$. For each replica $i$, stochastic parameters have to be identified. The pitch between two abscissae $x_{j}, x_{j+1}$ is denoted as $u_{j}$. The parameters $m_{Y i}, \sigma_{Y i}{ }^{2}, R_{Y i}\left(u_{k}\right)$ and $C_{Y i}\left(u_{k}\right)$ can be calculated as:

$$
\left\{\begin{array}{c}
m_{Y i}=\frac{1}{N} \sum_{j=1}^{N} y\left(x_{j}\right) \\
\sigma_{Y i}{ }^{2}=\frac{1}{N} \sum_{j=1}^{N} y\left(x_{j}\right)^{2}-m_{Y i}{ }^{2} \\
R_{Y i}\left(u_{k}\right)=\frac{1}{N-k} \sum_{j=1}^{N-k} y\left(x_{j}+u_{k}\right) y\left(x_{j}\right) \\
C_{Y i}\left(u_{k}\right)=R_{Y i}\left(u_{k}\right)-m_{Y i}{ }^{2}
\end{array}\right.
$$

Using Equations (4), the stochastic parameters can be identified from the experimental measurements (see Section 2). Based on the assumptions described above, the trajectory process is totally defined by the mean $m_{Y i}$, the standard deviation $\sigma_{Y i}$ and the autocorrelation function $C_{Y i}\left(u_{k}\right)$. The mean $m_{Y i}$ and the standard deviation $\sigma_{Y i}$ are calculated from (4). The 
autocorrelation function $C_{Y i}\left(u_{k}\right)$ has to be modelled. For this purpose, a negative exponential function $f^{\text {cor }}$ is commonly used:

$$
C_{Y i}\left(u_{k}\right) \approx f^{c o r}(u)=e^{-\frac{u}{\lambda_{i}}}
$$

where $u$ is the distance between two measurement points of the bond and $\lambda_{\mathrm{i}}$ is a length parameter.

\subsection{Identification of $Y(x, \omega)$ parameters}

The experimental field is developed first following (3) leading to trajectory fields $Y_{i}\left(x, \omega=\omega_{i}\right)$ with a mean equal to 0 . The decomposition is represented in Figure 3 . The identification procedure is applied to trajectory fields $Y_{i}\left(x, \omega=\omega_{i}\right)$ in order to identify the mean $m_{Y}$, the standard deviation $\sigma_{Y}$ and $\lambda$. For the identification of the $\lambda_{i}$-value, a least squares-based method is used. As explained above, we consider the stochastic field as 37 replicas. The stochastic parameters of $Y(x, \omega)$ are calculated as follows:

$$
\left\{\begin{array}{c}
m_{Y}=\frac{1}{37} \sum_{i} m_{Y i}=0 \mathrm{~m} \\
\sigma_{Y}=\frac{1}{36} \sum_{i} \sigma_{Y i}=7.410^{-6} \mathrm{~m} \\
\lambda=\frac{1}{37} \sum_{i} \lambda_{i}=1.3910^{-3} \mathrm{~m}
\end{array}\right.
$$

The ratio between the standard deviation and the mean value of the identified parameters characterizes the variability of the results. This ratio, the coefficient of variation (c.o.v.), is chosen to control the result quality. C.o.v. of the standard deviation (respectively of $\lambda$ ) is equal to 0.2 (respectively 0.3 ). Note that we have found similar results in the $y$-direction. This signifies that the stochastic field $Y(x, y, \omega)$ has isotropic properties. The identification of the mean $m_{Y}$ and the standard deviation $\sigma_{Y}$ is very well known. However, the identification stability of the $\lambda$-value has to be validated. Especially, we have to check that a c.o.v. equal to 0.3 does not lead to a significant error in the $\lambda$-estimate. In order to check the $\lambda$-identification process stability, a sensitivity analysis is done.

\section{$3.3 \lambda$-identification process stability}

To validate the $\lambda$-identification process stability, we build first a synthetic sample data set generated from $Y(x, \omega)$ as it is identified: $64 \times 37$ points (leading to 37 replicas of 64 points), a pitch equal to $1.1 \mathrm{~mm}, L=70 \mathrm{~mm}$. The identified stochastic parameters are also used in the sample data: $m_{Y}=0 \mathrm{~m}$ and $\sigma_{Y}=7.410^{-6} \mathrm{~m}$. However, for the $\lambda$-value, we generate sample 
data with 20 different values of $\lambda$ uniformly distributed in the range from $0.3 \mathrm{~mm}$ to $6 \mathrm{~mm}$. As explained above, for a given $\lambda$-value, c.o.v. of the identified parameter $\lambda$ characterizes the variability in the results and the result quality. The c.o.v. directly depends on 3 parameters: the length $L$, the pitch $u$ and the $\lambda$-estimate. Therefore, a sensitivity analysis of the c.o.v. is done with respect to the ratio $\frac{L}{\lambda}$ and the ratio $\frac{u}{L}$. The calculated c.o.v. of the $\lambda$-estimate is plotted according to these ratios in Figure 4. An "acceptable" zone is defined in the figure. It corresponds to an error less than $15 \%$ in the $\lambda$ value. This constraint corresponds to a c.o.v. less than 0.6. Point A represents the experimental characteristics of our problem $(u=1.1 \mathrm{~mm}$, $L=70 \mathrm{~mm}, \lambda=1.39 \mathrm{~mm}$ ). The c.o.v. of the $\lambda$-estimate calculated from Equation (6) is equal to 0.3 which satisfies the criterion.

Now the stochastic parameters of the adhesive thickness field have been identified and validated by the stability of the $\lambda$-identification process. They are now integrated in a reliability model.

\section{A combined mechanical and reliability model}

\subsection{Mechanical model}

Some classical models have been developed under some simple assumptions by Volkersen [4] or Hart-Smith [7] for instance. More complex models can be used in order to model non-linear behavior. In this first approach, it has been decided to choose a commonly used model developed by Volkersen [4] in order to highlight the feasibility of the proposed approach. The differential equations which govern the bonded joint behaviour read as follows:

$$
\left\{\begin{array}{c}
\frac{d^{2} \sigma_{x x}^{p}(x)}{d x^{2}}-\gamma \sigma_{x x}^{p}(x)=\beta \\
\sigma_{x z}^{a}(x)=e_{p} \frac{d^{2} \sigma_{x x}^{p}(x)}{d x^{2}}
\end{array}\right.
$$

with:

$$
\left\{\begin{array}{c}
\gamma=\frac{G_{a}}{e_{a}}\left(\frac{1}{e_{p} E_{x}}+\frac{1}{e_{s} E_{s}}\right) \\
\beta=-\frac{G_{a}}{e_{a} e_{p} E_{s}} \sigma_{x x}^{\infty}
\end{array}\right.
$$

$\sigma_{x x}^{p}(x), e_{p}$ and $E_{x}$ are, respectively, the longiditudinal stress component, the thickness and the Young's modulus of the bonded composite. $\sigma_{x z}^{a}(x), e_{a}$ and $G_{a}$ are, respectively, the shear stress component, the thickness and the shear modulus of the adhesive. $e_{s}$ and $E_{s}$ are, 
respectively, the thickness and the Young's modulus of the substrate. The substrate is subjected to a tensile stress $\sigma_{x x}^{\infty}$ in the $x$-direction. This differential equation may be solved analytically [14] when the thickness is constant. However, as explained above, the adhesive thickness variations are modelled leading to a non-uniform adhesive thickness distribution. We have, therefore, used a finite difference model to solve this differential equation.

\subsection{Stochastic model}

A stochastic model is developed here to take into account the uncertain spatial variations in the adhesive thickness. For this purpose, the adhesive thickness is modelled by a spline curve with nine interpolation points. The abscissae of these points are distributed according to a geometric distribution (see Figure 5). The mean value of the adhesive thickness is determined by the decomposition of the stochastic field (see Section 2.2), and it corresponds to $t^{\text {mean }}(x)$. These nine thicknesses $X_{i}$ represent the variables of the problem that constitute the vector $X$ with the stochastic characteristics identified in section 3 . These variables follow a lognormal distribution. The value of shear stress peak in the adhesive $\sigma_{x z}^{a}(0)$ enables us to evaluate the bonded joint failure through a limit state which is defined in the next section.

\subsection{Reliability model}

The stochastic parameters defined in equation (6) are integrated in a reliability model. The limit state $G(X)=0$ characterizes the bonded joint failure. It is written as follows:

$$
G(X)=S^{a}-\sigma_{x z}^{a}(0)
$$

The shear strength $S^{a}$ of the adhesive here is equal to $40 \mathrm{MPa}$. The shear stress in the adhesive is calculated with (7). There is failure of the bonded joint when $G(X) \leq 0$. The problem is then to calculate the probability to have $G(X) \leq 0$. The First Order Reliability Method (FORM) here is used [14-15]. It consists in an isoprobabilistic transformation, which transforms the random vector $X$ in the physical space to a random vector $U$ in the standard Gaussian space where the image of limit state is denoted as $H(U)=0$. The minimal distance between the origin and the limit state $H(U)=0$ represents the reliability index $\beta$. The closest point on the limit state represents the design point $U^{*}$. A failure probability $P_{f}$ is then approximated from the index $\beta$ :

$$
P_{f} \approx \Phi(-\beta)
$$

$\Phi$ represents the standard normal distribution. Determination of the index $\beta$ poses the constrained optimization problem:

$$
\beta^{2}=\min \|U\|^{2} \text {, subject to } H(U) \leq 0
$$


The optimization gives the reliability index $\beta$ and the failure probability $P_{f}$ through Equation (10). For this purpose, the reliability software FERUM v4.0 toolbox is used [18]. Note that the Second Order Reliability Method (SORM) has been used too and results are very close to results calculated with the FORM.

\section{Application}

\subsection{Determination of the failure probability}

Simulations are done with the stochastic parameters calculated in Section 3. We consider that the substrate is subjected to a tensile test with a stress equal to $110 \mathrm{MPa}$. We consider two types of models in order to highlight the influence of uncertain variations in adhesive thickness: the deterministic model and the stochastic model. The deterministic model takes into account the variability of the adhesive thickness without uncertain variations. In this case, the adhesive thickness is equal to $t^{\text {mean }}(x)$. The stochastic model takes into account uncertain variations. In this case, the adhesive thickness is equal to $t^{a}(x, \omega)$. Using the deterministic model, the shear stress peak can be calculated and is equal to $31.3 \mathrm{MPa}$, which corresponds to $78 \%$ of the strength $S^{a}$ of the adhesive. Using the stochastic model, the failure probability is equal to $83 \%$. We have a significant failure probability although a deterministic calculation equal only to $78 \%$ of the adhesive strength $S^{a}$. This important result clearly shows that the failure probability of the bonded joint is very high $(83 \%)$ when we consider the uncertain variations in the adhesive thickness despite good results in the deterministic case $\left(78 \%\right.$ of the strength $\left.S^{a}\right)$. Figure 6 represents the importance factors defined as follows:

$$
I=\frac{\alpha^{T} J_{U, x} D}{\left.\| \alpha^{T} J_{U, x} D\right) \|}
$$

$J_{U, x}$ represents the Jacobian of the isoprobabilistic transformation. $D$ represents the diagonal matrix of $\sqrt{J_{U, x} J_{U, x}^{T}}$. The $\alpha$-vector is defined as:

$$
\alpha=-\frac{\nabla_{U} H\left(U^{*}\right)}{\left\|\nabla_{U} H\left(U^{*}\right)\right\|}
$$

For the calculation of the importance factors, the reliability software FERUM is used [18]. The importance factors do not have a symmetric distribution. Indeed, they are very important near the right free edge. This is due to the fact that the adhesive works essentially near the free edge of the composite patch. So the adhesive thickness has no influence far away from the free edge. Moreover, the mean adhesive thickness is lower near the right free edge of the joint. So the uncertain variations in the adhesive thickness have more influence on this part of the joint. 


\subsection{Determination of safety coefficient}

The aim of this section is to determine a safety coefficient in order to have a failure probability lower than $0.01 \%$. Safety coefficient is expressed as a multiplication factor of the adhesive thickness. The idea is to change the initial setting of the adhesive thickness during the joint fabrication process to account for uncertain variations in the adhesive thickness. Calculations are done with safety coefficient $C s$ integrated in the mechanical model. For this purpose, simulations are done with a safe adhesive thickness $e_{a}^{C_{S}}(x)$ equal to:

$$
e_{a}^{C_{S}}(x)=C_{s} e_{a}(x)
$$

Figure 7 represents the failure probability according to Cs. This figure enables us to analyze the evolution of the failure probability as a function of Cs. value. The failure probability is very high when $C s$ is lower than 1 and decreases significantly to reach 1.2 . Afterwards, this coefficient is determined so as to have a failure probability less than $0.01 \%$. In this case, $C s$ is equal to 1.54 . This signifies that the initial adhesive thickness setting must be multiplied by 1.54 i.e., an adhesive characteristic thickness equal to $0.23 \mathrm{~mm}$ instead of $0.15 \mathrm{~mm}$.

\section{Conclusion}

This work highlights the influence of adhesive thickness variability on the failure of bonded joint. Measurements are done using a three-dimensional measuring machine and experimentally show this variability. An identification procedure enables us to have access to the stochastic parameters of the experimental adhesive thickness field. These parameters are identified and the stability of the identification process is checked. These identified parameters are integrated into a reliability model from which a failure probability of the bonded joint is calculated. Finally, the reliability model is used to determine a safety coefficient to decrease the failure probability to $0.01 \%$. The future of this study lies in improving the mechanical models. In particular, the thickness of the composite adherend can be progressively reduced near the end to reduce the shear stress amplitude. Furthermore, the geometry of the adhesive layer at the free edge ("square end" edges, spew fillets) can be taken into account too. This means that the solution in this more realistic case can probably only be carried out with a numerical model such as a finite element model, despite the problems which are generally encountered when modeling bonded joints with such a tool.. Moreover, surface approximation method may be used in order to increase the accuracy of the failure probability calculation, especially if finite element models are used.

\section{Acknowledgement}

The "Atelier Industriel de l'Aéronautique de Clermont-Ferrand" is gratefully acknowledged for its support during this study.

\section{REFERENCES}


Author produced version of the paper published in Journal of Adhesion Science and technology, vol. 27 (10), p. 1069-1079., 2013 Original publication available at http://www.tandfonline.com,

DOI : $10.1080 / 01694243.2012 .727176$

[1] L. Hollaway and M. Leerning, "Strengthening of reinforced concrete structures using externally-bonded FRP composites in structural and civil engineering", Woodhead PublishingLtd, Cambridge, (1999).

[2] A.A. Baker, Composite Structures, 2, 153-164, (1984).

[3] M.J Davis, in: Proceeding of the 3rd. Int. Workshop on Repair of Metallic Structures using Composites, 4, 1-15, (1995).

[4] O. Volkersen, Luftfahrtforschung, 15, 41-47, (1938).

[5] M. Tsai and J. Morton, Composite Structures, 32, 123-131, (1995).

[6] D. Oplinger, Int1. J. Solids Structures, 31, 2565-2587, (1994).

[7] L. Hart-Smith, “Adhesive-bonded single-lap joints”, Tech. Rep. CR-112236, NASA, (1973).

[8] L. Hart-Smith, "Adhesive-bonded double-lap joints", Tech. Rep. CR-112235, NASA, (1973).

[9] D. Bigwood and A. Crocombe, Intl. J. Adhesion Adhesives, 10, 31-41, (1990)

[10] P. Majda and J. Skrodzewicz, Intl. J. Adhesion Adhesives; 29, 396-404, (2009)

[11] G. Ji, Z. Ouyang, G. Li, S. Ibekwe and S.-S. Pang, Intl. J. Solids Structures, 47, 2445$2458,(2010)$

[12] R. Kahraman, M. Sunar and B. Yilbas, Mater. Process. Technol., 205, 183-189, (2008)

[13] A. A. Taib, R. Boukhili, S. Achiou, S. Gordon and H. Boukehili, Intl. J. Adhesion Adhesives, 26, 226-236, (2006)

[14] M. Lemaire, "Structural Reliability", John Wiley \& Sons, (2009)

[15] O. Ditlevsen, and H.O. Madsen, "Structural Reliability Methods", John Wiley and Sons, (1996)

[16] C.G. Bucher and U. Bourgund, Structural Safety, 7, 57-66, (1990).

[17] L. Bing, Z. Meilin and X. Kai, Reliability Eng. .System Safety, 67, 311-315, (2000). 
Author produced version of the paper published in Journal of Adhesion Science and technology, vol. 27 (10), p. 1069-1079., 2013 Original publication available at http://www.tandfonline.com,

DOI : $10.1080 / 01694243.2012 .727176$

[18] J.-M. Bourinet, C. Mattrand and V. Dubourg, in: Proceedings of the 10th International Conference On Structural Safety and Reliability (ICOSSAR 2009), Osaka, Japan, (2009). 
Author produced version of the paper published in Journal of Adhesion Science and technology, vol. 27 (10), p. 1069-1079., 2013 Original publication available at http://www.tandfonline.com,

DOI : $10.1080 / 01694243.2012 .727176$

Table 1 - Mechanical properties of the materials ${ }^{1}$.

\begin{tabular}{|c|c|c|c|c|c|}
\hline & $\begin{array}{c}E_{x} \\
(\mathrm{GPa})\end{array}$ & $\begin{array}{c}E_{y} \\
(\mathrm{GPa})\end{array}$ & $\begin{array}{c}v_{x y} \\
(-)\end{array}$ & $\begin{array}{c}G_{x y} \\
(\mathrm{GPa})\end{array}$ & $\begin{array}{c}S \text { (shear strength) } \\
(\mathrm{MPa})\end{array}$ \\
\hline Composite & 141 & 10 & 0.28 & 7 & 80 \\
\hline Aluminium & 70 & - & 0.32 & - & - \\
\hline Adhesive & 4.2 & - & 0.3 & - & 40 \\
\hline
\end{tabular}

\footnotetext{
${ }^{1} E_{x}$ (respectively $E_{y}$ ) corresponds to the Young's modulus in the $x$-direction (respectively in the $y$-direction). $v_{x y}$ represents the Poisson's ratio in the plane $(x, y) . G_{x y}$ represents the shear modulus in the plane $(x, y)$.
} 
Author produced version of the paper published in Journal of Adhesion Science and technology, vol. 27 (10), p. 1069-1079., 2013 Original publication available at http://www.tandfonline.com,

DOI : $10.1080 / 01694243.2012 .727176$

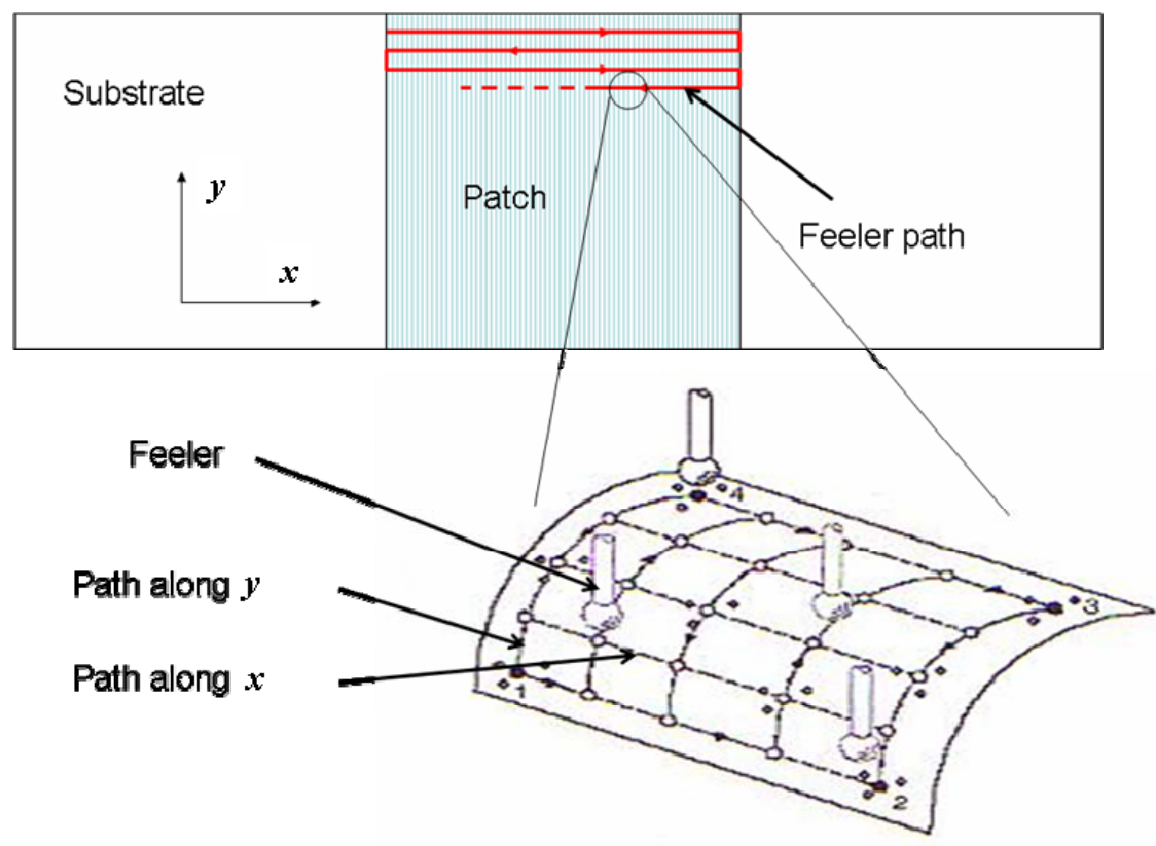

Fig. 1 Schematic view of the specimen and the path of the feeler. 


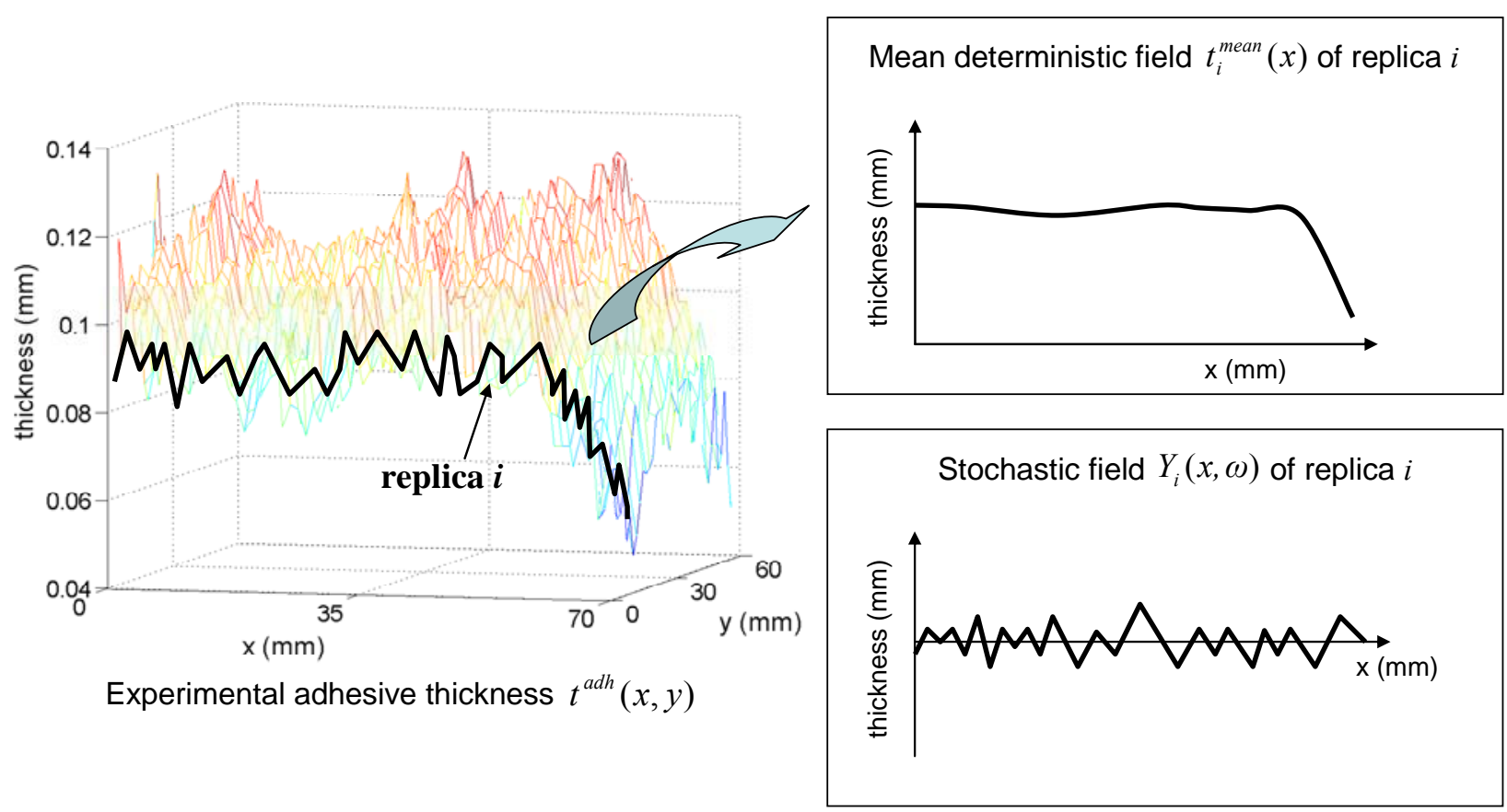

Fig. 2 Scheme of the decomposition of the experimental thickness $t^{a d h}(x, y)$. The experimental thickness $t^{a d h}(x, y)$ presents two types of variations: the first is global and is represented by the mean deterministic field $t_{i}^{\text {mean }}(x)$ for each measure in the $x$-direction; the second is modelled by a stochastic field $Y_{i}(x, \omega)$ for each measure in the $x$-direction. 


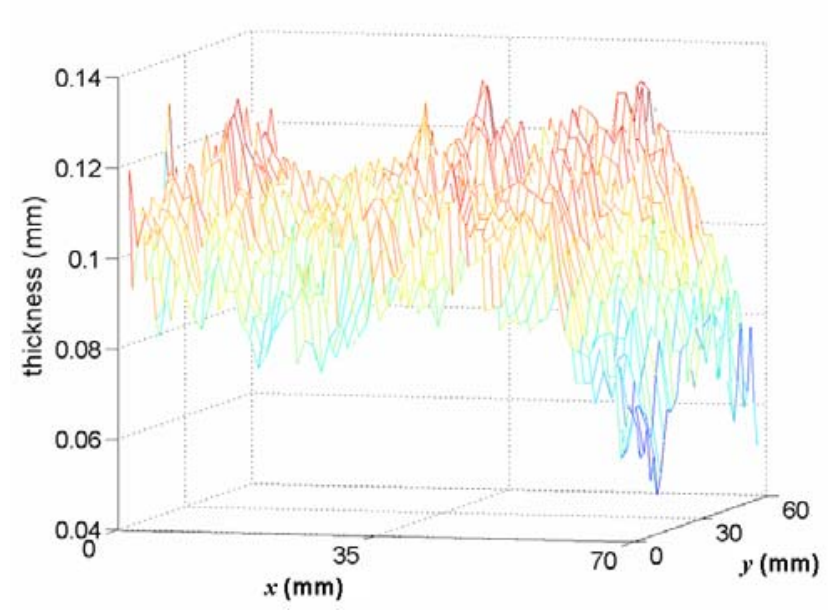

a - Total adhesive thickness $t^{a d h}(x, y)$

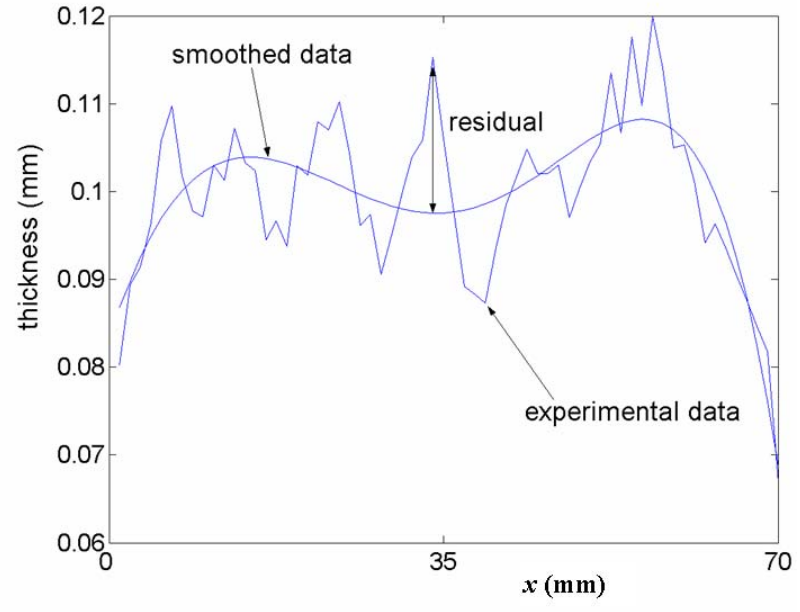

$\mathrm{b}$ - Smoothing example of a replica $t_{i}^{a}(x, \omega)$

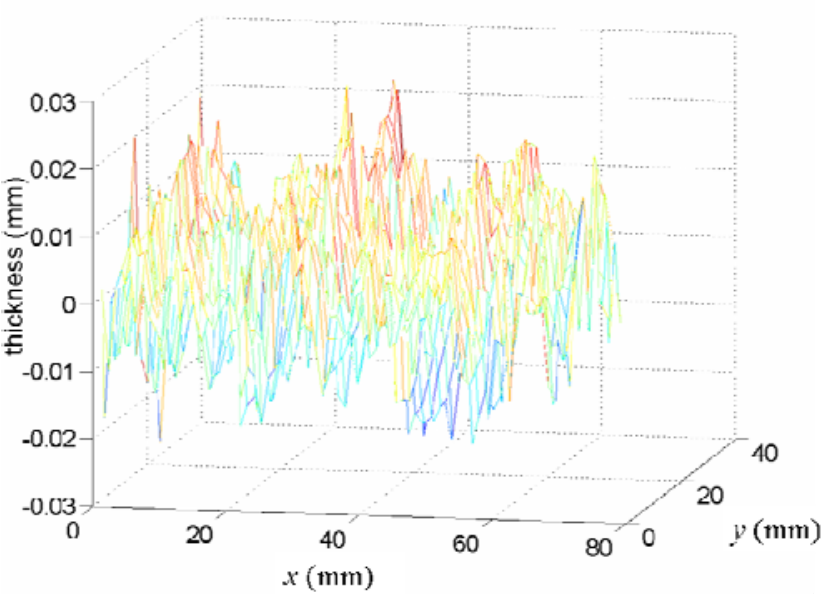

$\mathrm{d}$ - Stochastic fields $Y_{i}(x, \omega)$

Fig. 3 Results of the adhesive thickness field decomposition $t^{a d h}(x, y)$. Each measure $t_{i}^{a}(x, \omega)$ in the $x$-direction is smoothed in order to obtain the mean fields $t_{i}^{\text {mean }}(x)$. Then, the stochastic fields $Y_{i}(x, \omega)$ are obtained by subtracting the mean fields $t_{i}^{\text {mean }}(x)$ from the adhesive field $t^{a d h}(x, y)$. 
Author produced version of the paper published in Journal of Adhesion Science and technology, vol. 27 (10), p. 1069-1079., 2013 Original publication available at http://www.tandfonline.com,

DOI : $10.1080 / 01694243.2012 .727176$

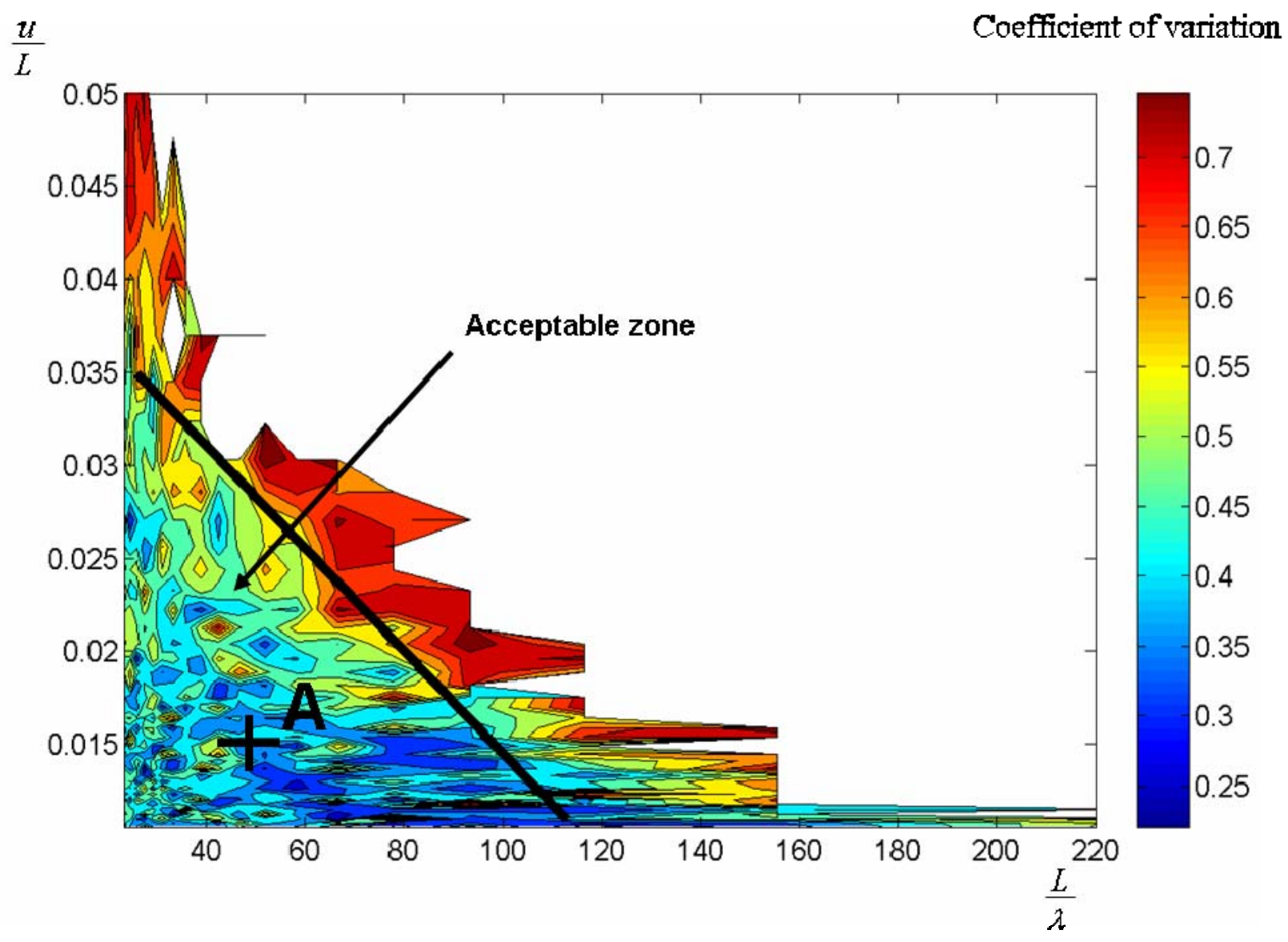

Fig. 4 Coefficient of variation according to $\frac{L}{\lambda}$ and $\frac{u}{L}$. 


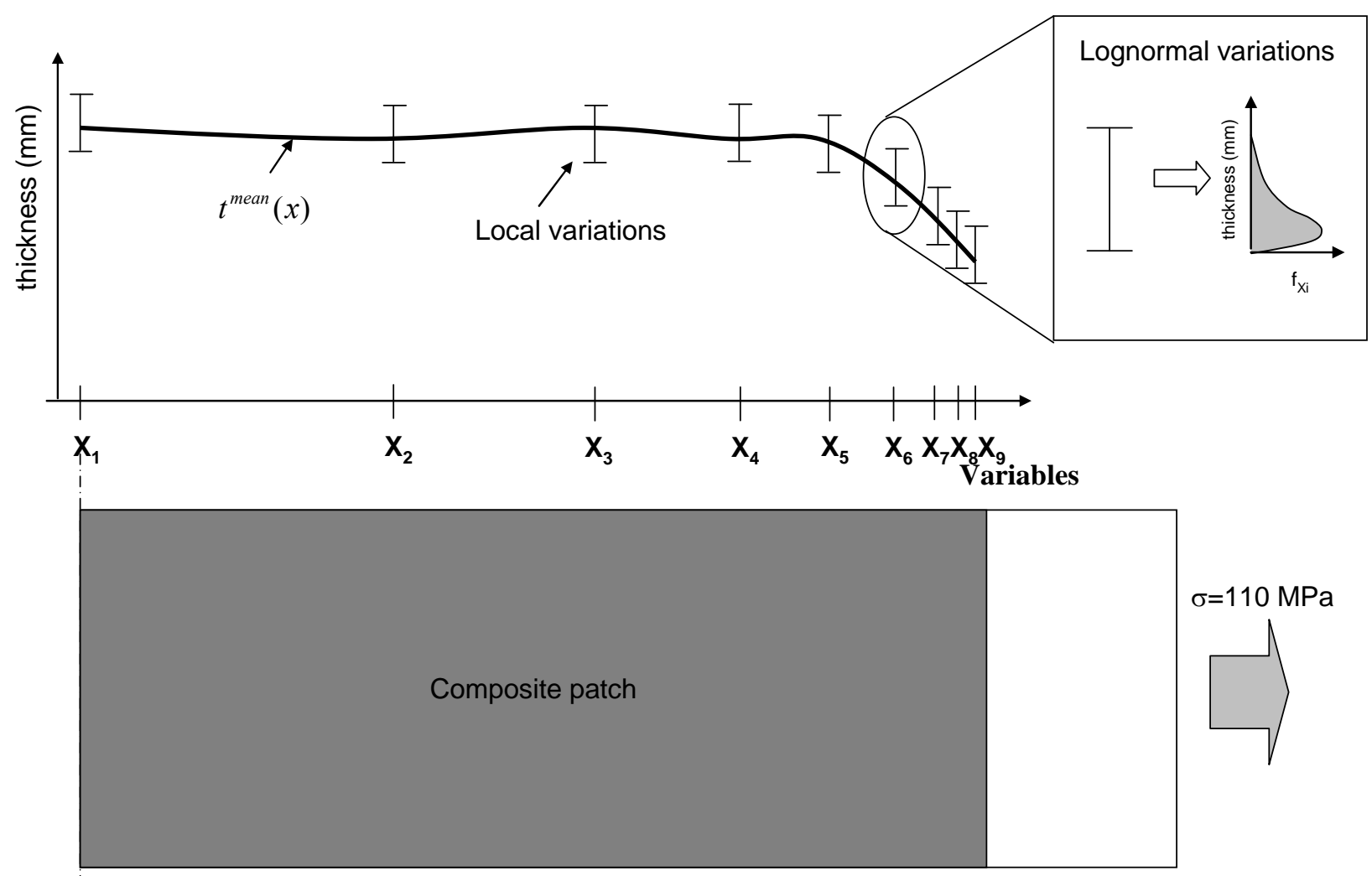

Fig. 5 Stochastic model: variables $X_{i}$ are considered to model variations in the adhesive thickness. These variables follow a lognormal distribution and their abscissae are distributed according to a geometric distribution. 
Author produced version of the paper published in Journal of Adhesion Science and technology, vol. 27 (10), p. 1069-1079., 2013 Original publication available at http://www.tandfonline.com,

DOI : $10.1080 / 01694243.2012 .727176$

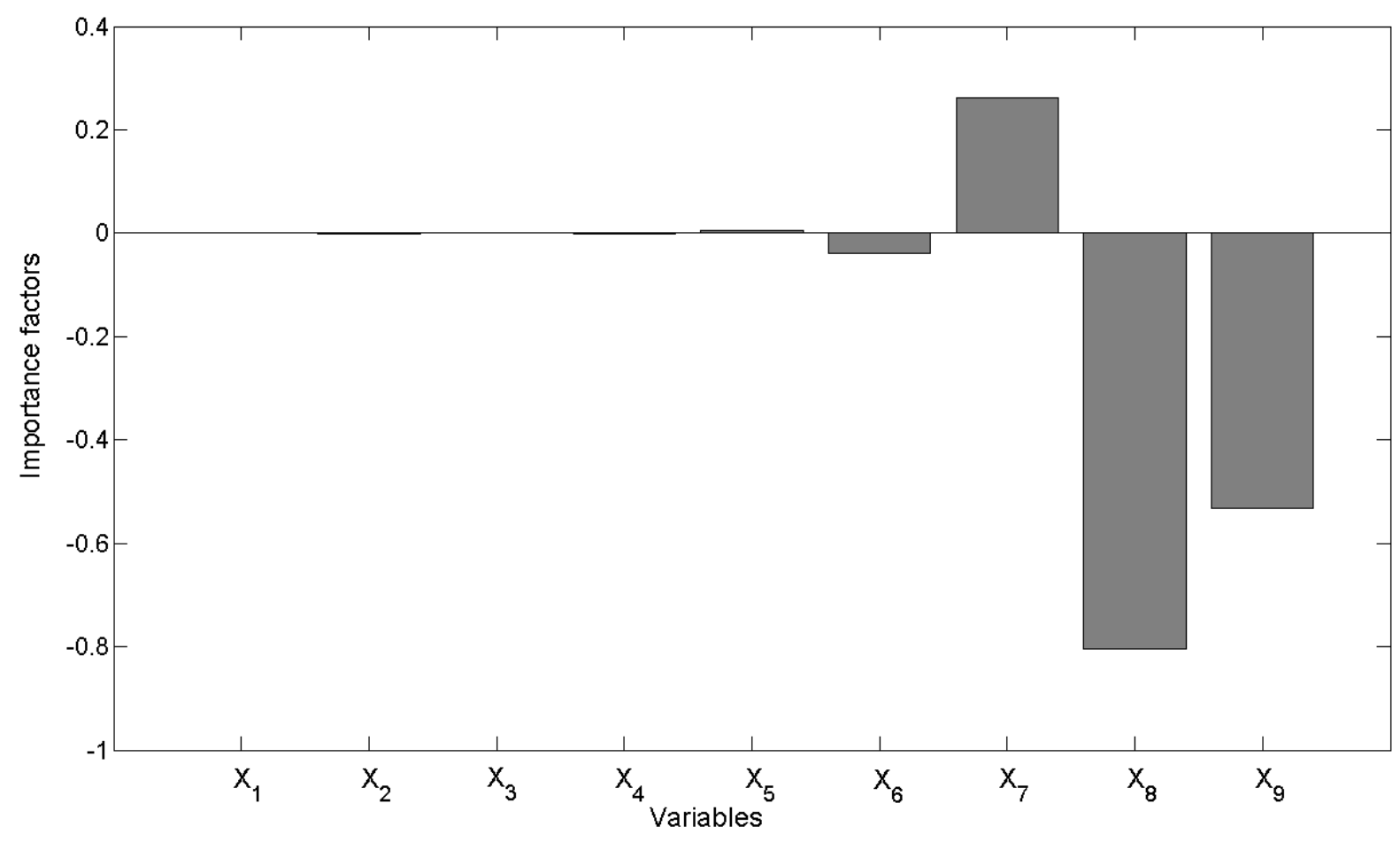

Fig. 6 Importance factors of the $X_{i^{-}}$variables. The variables located near the free edge $\left(X_{7}\right.$, $\left.X_{8}, X_{9}\right)$ have significant importance factors. Therefore, their influences on the failure probability of the bonded joint are higher than the influences of the other variables. 
Author produced version of the paper published in Journal of Adhesion Science and technology, vol. 27 (10), p. 1069-1079., 2013 Original publication available at http://www.tandfonline.com,

DOI : $10.1080 / 01694243.2012 .727176$

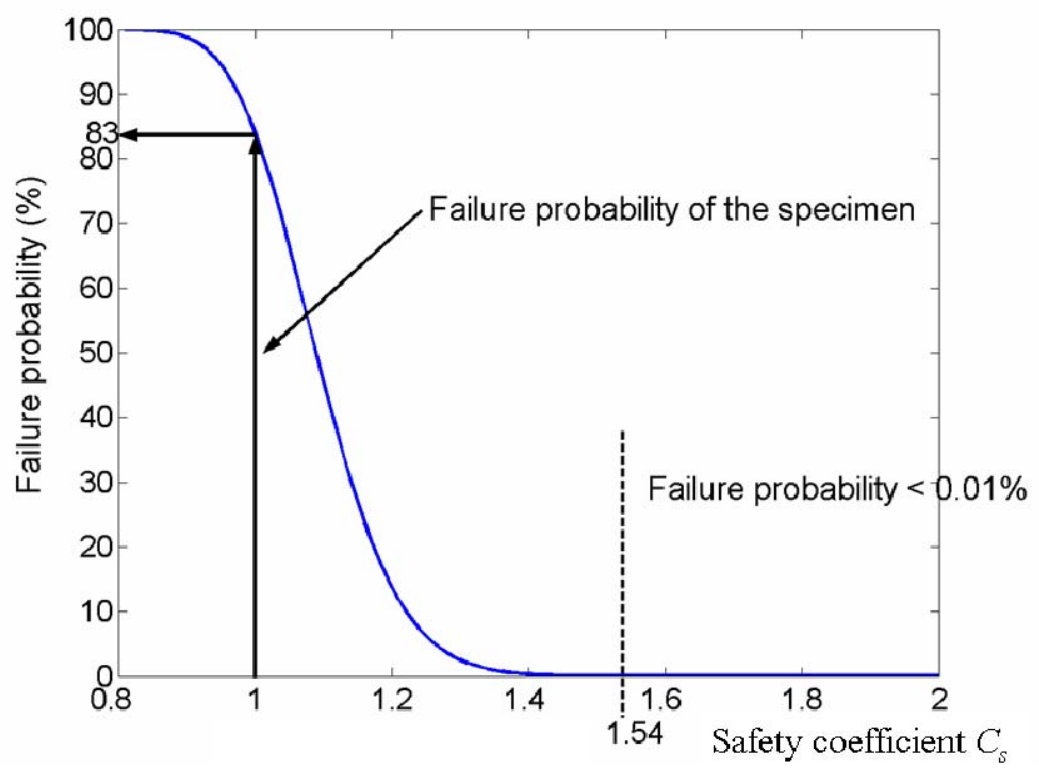

Fig. 7 Failure probability according to the safety coefficient $C_{s}$. 